\title{
CONTROL OF DISCRETE-TIME PIECEWISE AFFINE SYSTEMS
}

\author{
Thomas Erhard Hodrus, Michael Buchholz, Volker Krebs
}

\author{
Institut für Regelungs- und Steuerungssysteme, \\ Universität Karlsruhe (TH), Germany \\ phone: $+49721 / 6082462$ \\ fax: $+49721 / 6082707$ \\ e-mail: $\{$ hodrus,krebs\}@irs.uni-karlsruhe.de
}

\begin{abstract}
In this paper we propose a new approach for the control of discrete-time piecewise affine hybrid systems on full-dimensional polytopes. The new strategy is divided into a local and a supervisory control problem. The local problem is to reach and cross one facet of a polytope ensuring that the next sample of the time-discrete trajectory is picked up in the adjacent polytope. Therefore, a local piecewise affine control law is obtained by solving an optimization problem, minimizing the retention period of the trajectory in the polytope or the quadratic sum of the input signal. This procedure is based on system inherent bounds. The supervisory control problem is to find a suitable combination of polytopes and local control strategies that transfers the trajectory to the operating point and keeps it there.
\end{abstract}

Copyright (C)2005 IFAC

Keywords: hybrid systems, affine systems, polytope, discrete-time control

\section{INTRODUCTION}

The piecewise linear approach for nonlinear systems was proposed in (Sontag, 1981). This approach has been generalized and finally led to hybrid systems theory. In (Branicky, 1995) the hybrid phenomena were formulated. After this milestone a lot of research has been done, especially in modeling of hybrid systems. The analysis of this class of systems and the synthesis of controllers are still sophisticated, even nowadays.

There are two different directions in literature for the design of controllers for hybrid and piecewise affine systems. In (Nenninger, 2001; Habets and van Schuppen, 2004) continuous time controllers and in (Bemporad and Morari, 1999; Borrelli, 2002; Imura, 2004) discrete-time controllers are designed.
Discrete-time affine systems can be obtained from measurements of technical processes by identification (Münz and Krebs, 2002; Ferrari-Trecate, 2001) of the hybrid system or by theoretical modeling (Hodrus and Münz, 2003; Hodrus et al., 2004). In the theoretical modeling approach, given continuous piecewise affine models are discretized calculating the discrete-time affine model in each domain from the valid continuous model.

In this paper, a new approach will be presented for the control of discrete-time piecewise affine systems that represent a sub class of hybrid systems.

In section 2 some basic notions as polytope, facet, affine system, and affine control law are given. Using this terms, the problem of dynamical systems restricted to a polytope is presented. The simplex 
as a special polytope is introduced. The aim of the local controller design is formulated.

System inherent bounds, on which the new controller design is based, are given in section 3

In section 4 , the new control strategy is developed. It consists of a supervisory and a local control strategy. At the beginning, the considered state space is partitioned into a simplex structure. Each simplex has a corresponding vertex in a graph. The local control strategy completes the graph by adding selected edges. After the local control is explored, the completed graph is used for supervisory control.

The task of the supervisory control is to find a path through the graph structure. The local and the supervisory control strategy are calculated off-line. The online effort is to determine the active simplex and therefore, the active control law, solving a linear search problem. This new approach is able to track very fast dynamics because of the reduced on-line effort.

Finally, the new strategy is applied to a two tank system in section 5 . The two tank system can be modeled as a piecewise affine system with discrete and continuous input signals. A performance index will determine which piecewise affine dynamic assigned to a combination of discrete inputs is the best.

The contribution is finished with a conclusion in section 6 .

\section{PROBLEM STATEMENT}

The considered state space $\underline{x} \in \mathbb{R}^{N}, N \in \mathbb{N}$ is bounded on the set $\Theta$. Assuming there are $M$ points $\underline{v}_{1}, \ldots, \underline{v}_{M}$, with $M \geq N+1$, in state space $\mathbb{R}^{N}$, such that there exists no hyperplane of $\mathbb{R}^{N}$, containing all these $M$ points. The fulldimensional polytope $P$ is defined as the convex hull of $\underline{v}_{1}, \ldots, \underline{v}_{M}$. If a point $\underline{v}_{i},(i=1, \ldots, M)$ cannot be written as convex combination of the points $\underline{v}_{1}, \ldots, \underline{v}_{i-1}, \underline{v}_{i+1}, \ldots, \underline{v}_{M}$ it is called a vertex of the polytope $P$. The polytope is completely characterized by its set of vertices. A full-dimensional polytope with $M=N+1$ is called a full-dimensional simplex.

The intersection of a finite number of half spaces can also describe a polytope. If an integer $K \geq$ $N+1$, non-zero vectors $\underline{n}_{1}, \ldots, \underline{n}_{K} \in \mathbb{R}^{N}$, and scalars $\alpha_{1}, \ldots, \alpha_{K} \in \mathbb{R}$ exists, such that

$$
P=\left\{x \in \mathbb{R}^{N} \mid \forall i=1, \ldots, K: \underline{n}_{i}^{T} \underline{x} \leq \alpha_{i}\right\}
$$

is valid, then (1) is the implicit description of a polytope.

The intersection of a full-dimensional polytope $P$ with one of its supporting hyperplanes

$$
F_{i}=\left\{x \in \mathbb{R}^{N} \mid \underline{n}_{i}^{T} \underline{x}=\alpha_{i}\right\} \cap P
$$

is called a facet $F_{i}$ of $P$, if the dimension of the intersection is equal to $N-1$. By convention the vector $\underline{n}_{i}$, that is the normal vector of the facet $F_{i}$, is of unit length and points out of the polytope.

Using numbered vertices of a simplex, the facets can be named with the number of that vertex which is not a vertex of the facet.

In (Habets and van Schuppen, 2004) a method is presented to partition full-dimensional polytopes into full-dimensional simplices by using e.g. the Delaunay-triangulation.

Therefore, in the sequel, the full-dimensional simplex $P$ in $\mathbb{R}^{N}$ with $N+1$ facets is used.

On each full-dimensional simplex $P$ a discretetime affine system

$$
\underline{x}_{k+1}=\underline{\Phi}_{x_{k}}+\underline{H}_{u_{k}}+\underline{\phi}
$$

is considered, with $\underline{\Phi} \in \mathbb{R}^{N \times N}, \underline{H} \in \mathbb{R}^{N \times m}$ and $\phi \in \mathbb{R}^{N}$. The state $\underline{x} \in \mathbb{R}^{N}$ is assumed to be contained in the polytope $P$. The input signal $\underline{u}$ takes values from the bounded set $U \subset \mathbb{R}^{m}$ of continuous inputs.

The discrete-time piecewise affine system is defined on a subset $\Theta$ of the state space. There are several affine systems $i$, each defined on a simplex $P_{i} \subseteq \Theta$, with $\bigcup P_{i}=\Theta, P_{i} \cap P_{j}=\emptyset \quad \forall i \neq j$.

The aim of the controller design is to find discretetime affine control laws

$$
\underline{u}_{k}=-\underline{R}_{i} \underline{x}_{k}+\underline{u}_{0, i}
$$

defined on the simplex $P_{i}$, such that the trajectories of the controlled system

$$
\underline{x}_{k+1}=\left(\underline{\Phi}_{i}-\underline{H}_{i} \underline{R}_{i}\right) \underline{x}_{k}+\left(\underline{\phi}_{i}+\underline{H}_{i} \underline{u}_{0, i}\right),
$$

starting at any possible point in $P_{i}$

- will stay in the simplex $P_{i}$, or

- enter one of the $N+1$ adjacent simplices through one specific facet of the simplex.

The controlled system in equation (5) is equal to (3), with $\left(\underline{\Phi}_{i}+\underline{H}_{i} \underline{R}_{i}\right)=\underline{\Phi}_{R}$ and $\left(\underline{\phi}_{i}+\underline{H}_{i} \underline{u}_{0, i}\right)=$ $\underline{\phi}_{R}$. The result is an autonomous discrete-time affine system

$$
\underline{x}_{k+1}=\underline{\Phi}_{R} \underline{x}_{k}+\underline{\phi}_{R} .
$$

The following example shall clarify the main challenge of the controller design of discrete-time piecewise affine systems.

Example 1. (Trajectories in state space). In figure 1 a trajectory of a stable discrete-time affine system is presented. In the left part, the trajectory is starting at the initial state $\underline{x}_{0}$ and evolves to the stationary state $\underline{x}_{\infty}$. In the right part of figure $1, \mathrm{a}$ simplex structure was superimposed. It is obvious that the trajectory leaves simplex $P_{1}$ and only 

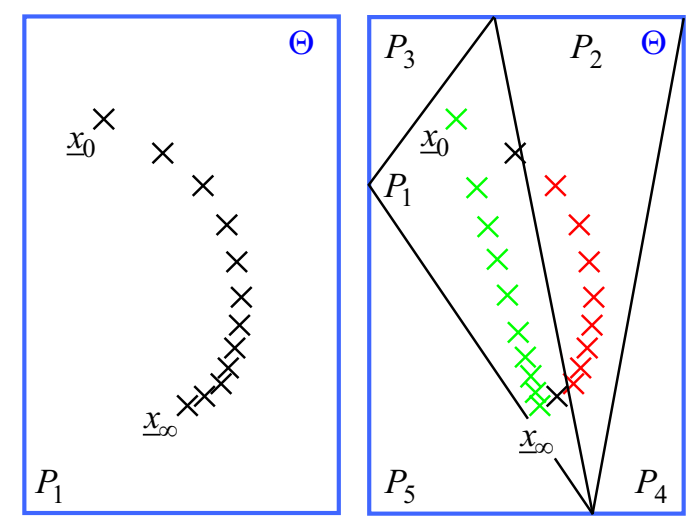

Fig. 1. Trajectory of a discrete-time affine system in state space

reaches the stationary state $\underline{x}_{\infty}$ if the dynamic in $P_{2}$ equals the dynamic in $P_{1}$.

In the right part, there is a second trajectory that stays in the simplex $P_{1}$. This is the result of an affine control law, as stated in (4), valid in $P_{1}$. Alternatively, the trajectory could be forced to leave the simplex through a selected facet.

If there are discrete inputs $\underline{u}_{D}$ to the system, $\underline{u}_{D}$ is an element of a bounded integer set, see details in (Nenninger, 2001) or section 5, a finite number of discrete-time piecewise affine systems can be determined for each possible combination of the elements of the finite discrete input vector $\underline{u}_{D}$. Let each combination be numbered serially in the variable $x_{D}$.

For each combination $x_{D}$ the same continuous state space $\Theta$ with the same partitioning into simplices is considered. There are $\max \left(x_{D}\right)$ dynamics defined for one simplex.

If the trajectory enters a simplex or is within the start simplex, this is a necessary condition, that the discrete input $\underline{u}_{D}$ changes.

In the next section, system inherent bounds are used to influence the system behavior.

\section{SYSTEM INHERENT BOUNDS}

Each autonomous system $\underline{x}_{k+1}=\underline{\Phi} \underline{x}_{k}$ has inherent system bounds. If the eigenvalues of the matrix $\underline{\Phi}$ are real valued and positive, these system inherent bounds represent hyperplanes crossing the origin of the system. In figure 2 a 2 dimensional case is shown, where the system inherent bounds are given by the straight lines $I_{1}$ and $I_{2}$. If the eigenvalues are complex, the system inherent bounds can be described with ellipsoids. The trajectory of a stable system, once entering the ellipsoid is trapped within (Nenninger, 2001). These complex eigenvalues are not topic of this paper.
If an affine term $\phi$ is added to the autonomous system, the equilibrium of the system leaves the origin. This leads e.g. to the controlled discretetime affine system (6).

The system inherent bounds cross the equilibrium $\underline{x}_{e}$, if the left eigenvectors $\underline{w}_{i}$ of the system matrix $\underline{\Phi}_{R}$ and the elements of the system matrix $\underline{\Phi}_{R}$ are real valued, that means that the eigenvalues $\lambda_{i}$ also have to be real valued.

The following equation $\underline{w}_{i} \underline{x}=\underline{w}_{i} \underline{x}_{e}$ describes the system inherent bounds, that are hyperplanes. System inherent bounds means that there exists no trajectory which is able to cross this hyperplane under the assumptions made.

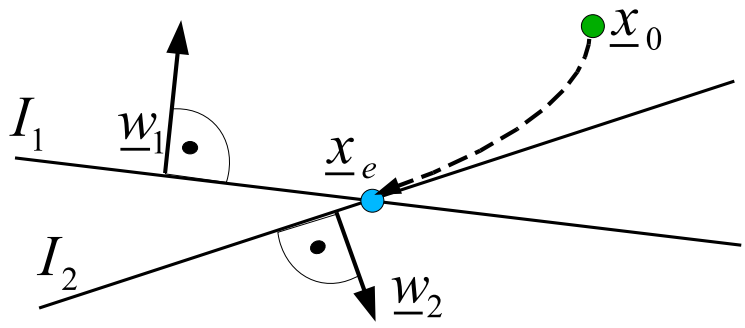

Fig. 2. The system inherent bounds $I_{1}$ and $I_{2}$ corresponding to the eigenvectors $\underline{w}_{1}$ and $\underline{w}_{2}$.

In figure 2 , the equilibrium $\underline{x}_{e}$ is given. The left eigenvectors $\underline{w}_{i}$ are normal vectors to the system inherent bounds $I_{1}$ and $I_{2}$, that are hyperplanes. The trajectory starting in $\underline{x}_{0}$ moves to the equilibrium $\underline{x}_{e}$, not able to cross the system inherent bounds $I_{1}$ and $I_{2}$.

For continuous time systems this result was proven in (Nenninger, 2001). Transferring his conclusions to discrete-time systems means to characterize the eigenvalues $\lambda_{i} \in\left\{\mathbb{R} \mid 0 \leq \lambda_{i}<1\right\}$ for a considered stable system.

For the intended controller design the left eigenvectors have to be linear independent.

In the next section, the system inherent bounds are used to design a local controller.

\section{CONTROL STRATEGY}

Given a discrete-time piecewise affine system in state space $\Theta$, partitioned into simplices, the relation between the numbered simplices can be stored in a graph structure. Each simplex $P$ is represented by a vertex in this graph. The control strategy consists of two parts.

First, the local control strategy will be investigated. Using system inherent bounds, the local strategy tries to find a discrete-time piecewise affine control law, ensuring that every trajectory, starting in simplex $P$ will leave the simplex 
through a specific facet $F_{i}, i \in 1, \ldots, N+1$ of the simplex $P$ and the sample picked up next is within the adjacent simplex $P_{A}$. The simplex $P$ and $P_{A}$ share the same facet $F_{i}$.

If a valid control law is found, an oriented edge is added to the graph, connecting simplex $P$ with $P_{A}$. This edge is used to store the two determined parameters $\underline{R}$ and $\underline{u}_{0}$ of the control law (4). If discrete inputs $\underline{u}_{D}$ exist, a third parameter would be $x_{D}$. The best possibility will be chosen minimizing a performance index in such a way that

- the retention time in the simplex $P$ is minimized or

- the control energy is minimized.

It is assumed that a change in $x_{D}$ is only allowed, if the first sample after the trajectory crossed a facet is picked up.

\subsection{Local control}

The local control problem, as mentioned in section 2 , is to enter the adjacent simplex $P_{A}$ through a defined facet $F_{A}$, if the state $\underline{x} \in \Theta \subset \mathbb{R}^{N}$ is not contained in the final simplex $P_{F}$. The sequence of simplices is determined by the supervisory control.

If the state $\underline{x}$ is in the final simplex, the local control problem ist to stabilize the state in $\underline{x}_{F}$ and keep the trajectory in $P_{F}$.

The control law that solves the problem, consists of two parameters $\underline{R}$ and $\underline{u}_{0}$. The aim of this procedure is to find a matrix $\underline{R}$ and the vector $\underline{u}_{0}$, such that constraints in the input signal $\underline{u}$ are satisfied.

For each facet of each simplex, the local control algorithm tries to find a control law (4). If there are discrete inputs $\underline{u}_{D}$, the effort for exploring all simplices is multiplied with $\max \left(x_{D}\right)$.

For the local control used to enter an adjacent simplex through one specific facet two different methods will be presented.

Let an operating point $\underline{x}_{\infty}$ in a region of the adjacent simplex $P_{A}^{*}$ be chosen. If $P \cup P_{A}$ results in a convex region, than $P_{A}^{*}=P_{A}$. As shown in figure $4 P_{A}^{*}$ can be a subregion of $P_{A}, P_{A}^{*} \subset P_{A}$, if $P \cup P_{A}$ is not convex.

The first method is presented in figure 3. After the $\underline{x}_{\infty}$ is selected, the system inherent bounds are given by straight lines through the point $\underline{x}_{\infty}$ and the vertices $v_{j}, j \in\{1, . ., i-1, i+1, . ., N+1\}$ of the facet $F_{i}$. In figure 3 e.g. $i=3$, the vertices $v_{1}$ and $v_{2}$ are used together with $\underline{x}_{\infty}$ to determine the system inherent bounds, the straight lines

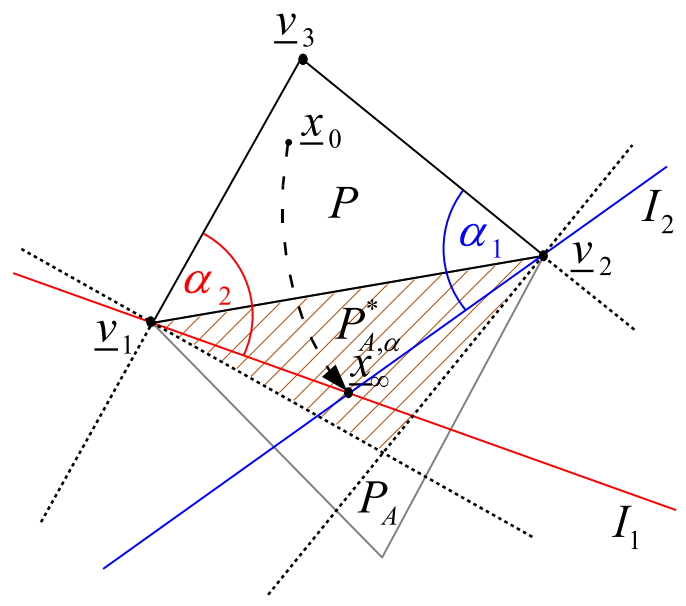

Fig. 3. The controlled system with real eigenvalues and selected eigenvectors.

$I_{1}=\left(\underline{v}_{1}, \underline{x}_{\infty}\right)$ and $I_{2}=\left(\underline{v}_{2}, \underline{x}_{\infty}\right)$. Additionally, $\underline{x}_{\infty}$ has to be chosen that $\alpha_{j} \leq 90^{\circ}$ with $P_{A, \alpha}^{*} \subseteq P_{A}^{*}$.

In this case, the two eigenvalues $\lambda_{1}$ and $\lambda_{2}$ do not have to be be equal, but both have to be stable. A sample trajectory, not a straight line for this general case, from $\underline{x}_{0}$ to $\underline{x}_{\infty}$ is given.

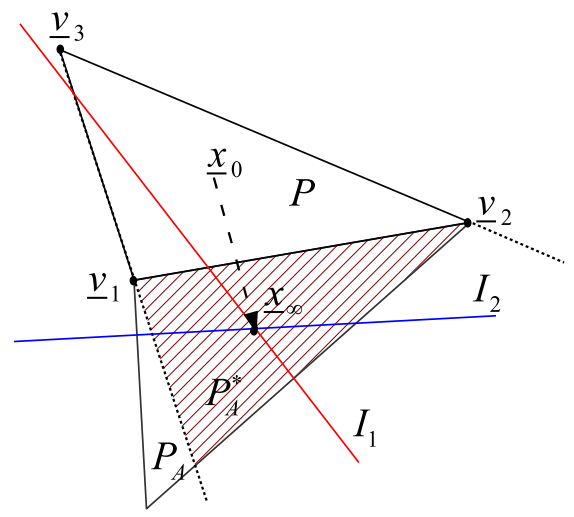

Fig. 4. The controlled system with multiple real stable eigenvalues, the operating point on a restricted area of the neighbor simplex.

The second method is to choose the $\underline{x}_{\infty} \in P_{A}^{*}$ and real multiple eigenvalues $\lambda_{i}=\lambda, i=1, \ldots, N$. In figure 4 an example is given with $\lambda_{1}=\lambda_{2}$. The trajectories are straight lines due to the multiple eigenvalues, an example is given from $\underline{x}_{0}$ to $\underline{x}_{\infty}$. The system inherent bounds $I_{1}$ and $I_{2}$ only have to be linearly independent.

Both methods can be formulated as a standard optimization problem, a so called constrained minmax-problem. A solver for this problem is e.g. fminimax contained in the MATLAB OptimizATION TOOLBOX.

The stabilizing control in the final simplex $P_{F}$ can be determined with the second method, mentioned to leave the simplex through a facet, with $\underline{x}_{\infty}=\underline{x}_{F}$ is in the simplex $P_{F}$. Formulating an 
optimization problem the parameter $\underline{R}$ and $\underline{u}_{0}$ can be determined.

\subsection{Supervisory control}

The local control provides a graph with edges. The supervisory control reduces to a problem to find a path in this graph. This is also a standard problem and can be solved with Dijkstra's-Algorithm (Nemhauser et al., 1989).

\section{EXAMPLE}

The system under investigation is shown in figure 5. For each tank there is an influx. The left tank is labeled with 1 and the right tank with 3 , and the influxes are labeled with $q_{1}$ and $q_{3}$, respectively. In table 1 the discrete state $x_{D}$ as

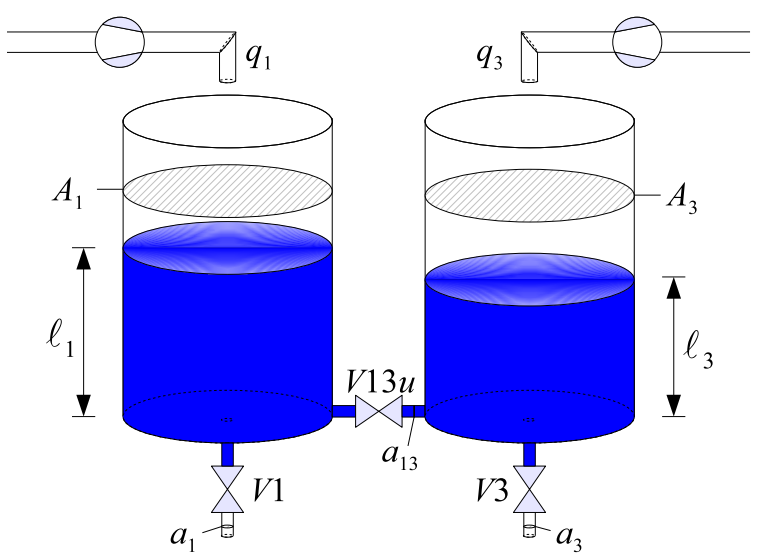

Fig. 5. Two tank system

Table 1. Combinations of the valves and discrete state $x_{D}$

\begin{tabular}{ccccccccc}
$x_{D}$ & 1 & 2 & 3 & 4 & 5 & 6 & 7 & 8 \\
\hline $\mathrm{V} 1$ & 0 & 0 & 0 & 0 & 1 & 1 & 1 & 1 \\
$\mathrm{~V} 3$ & 0 & 0 & 1 & 1 & 0 & 0 & 1 & 1 \\
$\mathrm{~V} 13 \mathrm{u}$ & 0 & 1 & 0 & 1 & 0 & 1 & 0 & 1
\end{tabular}

combinations of the discrete input vector $\underline{u}_{D}=$ $(V 1, V 3, V 13 u)$ is given. A valve is open, if the value of the binary variable is one, e.g. $V 1=1$. The dynamic for the two tank system for $x_{D}=8$ is

$$
\begin{aligned}
& \dot{\ell}_{1}(t)= \\
& -\operatorname{sign}\left(\ell_{1}(t)-\ell_{3}(t)\right) \frac{a_{13}}{A_{1}} \sqrt{2 g} \sqrt{\left|\ell_{1}(t)-\ell_{3}(t)\right|} \\
& -\frac{a_{1}}{A_{1}} \sqrt{2 g} \sqrt{\ell_{1}(t)}+\frac{q_{1, \text { max }}}{A_{1}} u_{q, 1}(t) \\
& \dot{\ell}_{3}(t)= \\
& -\operatorname{sign}\left(\ell_{1}(t)-\ell_{3}(t)\right) \frac{a_{13}}{A_{3}} \sqrt{2 g} \sqrt{\left|\ell_{1}(t)-\ell_{3}(t)\right|} \\
& -\frac{a_{3}}{A_{3}} \sqrt{2 g} \sqrt{\ell_{1}(t)}+\frac{q_{3, \max }}{A_{3}} u_{q, 3}(t) .
\end{aligned}
$$

The input $\underline{u}(t)=\left(u_{q, 1}(t), u_{q, 3}(t)\right)^{T}$ is added linearly to the nonlinear state equation. The state vector is $\underline{x}(t)=\left(\ell_{1}(t), \ell_{3}(t)\right)^{T}$. The root function in the state equation is approximated with a discrete-time affine system valid on a simplex $P$ for the controller design, simulation later on is done on the nonlinear system. In figure 6 , the partitioned state space $\Theta, \underline{x} \in\left\{\Theta \subset \mathbb{R}^{2} \mid 0 \leq\right.$ $\left.x_{1}=\ell_{1} \leq 60[\mathrm{~cm}], 0 \leq x_{2}=\ell_{3} \leq 60[\mathrm{~cm}]\right\}$ into simplices is given. The simplices are num-

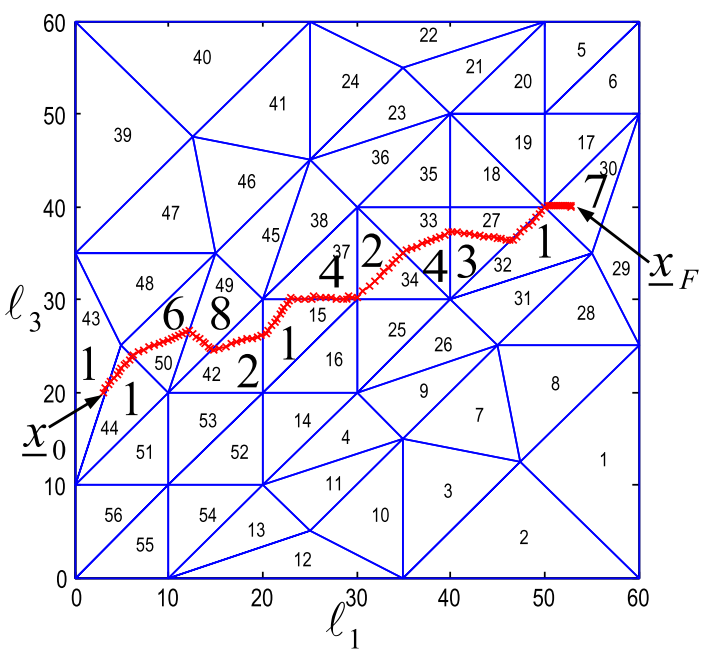

Fig. 6. The trajectory of the two tank system, starting in $\underline{x}_{0}=(3,20)[\mathrm{cm}]$ moving to $\underline{x}_{F}=$ $(53,40)[\mathrm{cm}]$. Sampling time $T=1 \mathrm{~s}$.

bered. The trajectory moves from the initial state $\underline{x}_{0}=(3,20)[\mathrm{cm}]$ to final state $\underline{x}_{F}=(53,40)[\mathrm{cm}]$ crossing the simplices summarized in table 2 . The information which valves are closed or opened, is given by $x_{D}$ marked with big digits in these twelve used simplices in figure 6 .

Table 2. Trajectory from $\underline{x}_{0}$ to $\underline{x}_{F}$ sequentially moves over the simplices $P_{i}$

\begin{tabular}{ccccccc} 
Step & $\underline{x}_{0}$ & 2 & 3 & 4 & 5 & 6 \\
\hline$P_{i}$ & 43 & 44 & 50 & 49 & 42 & 15 \\
$x_{D}$ & 1 & 1 & 6 & 8 & 2 & 1 \\
Step & 7 & 8 & 9 & 10 & 11 & $\underline{x}_{F}$ \\
\hline$P_{i}$ & 37 & 34 & 33 & 27 & 32 & 30 \\
$x_{D}$ & 4 & 2 & 4 & 3 & 1 & 7
\end{tabular}

For simplex '50' details are given

$$
\begin{aligned}
& \underline{\Phi}_{50}^{6}=\left[\begin{array}{ll}
0.9794 & 0.0095 \\
0.0088 & 0.9911
\end{array}\right], \underline{\phi}_{50}^{6}=\left[\begin{array}{c}
0.0142 \\
-0.1260
\end{array}\right] \\
& \text { and } \underline{H}_{50}^{6}=\left[\begin{array}{ll}
0.6429 & 0.0031 \\
0.0029 & 0.6467
\end{array}\right] \text {. }
\end{aligned}
$$

The upper index denotes the $x_{D}$ to indicate the necessary combination of the valves. The two parameters of an control are given:

$\underline{R}_{50 \rightarrow 49}^{6}=\left[\begin{array}{ll}0.0353 & 0.0146 \\ 0.0135 & 0.0532\end{array}\right], \underline{u}_{0,50 \rightarrow 49}^{6}=\left[\begin{array}{l}1.3156 \\ 2.1996\end{array}\right]$

The lower index denotes, where the trajectories start and where they will lead to, e.g. $50 \rightarrow 49$ 
means the control law is valid in simplex '50' and will force the trajectory to enter simplex ' 49 '. There were no control laws found for $\underline{R}_{50 \rightarrow 48}^{6}$ and $\underline{R}_{50 \rightarrow 44}^{6}$ that consider the limitation of the input vector $\underline{u}$. Other possible control laws were found for $\underline{R}_{50 \rightarrow 49}^{3}, \underline{R}_{50 \rightarrow 48}^{5}, \underline{R}_{50 \rightarrow 49}^{7}, \underline{R}_{50 \rightarrow 48}^{7}$ and $\underline{R}_{50 \rightarrow 44}^{7}$. The control law $\underline{R}_{50 \rightarrow 49}^{6}$ has the performance index $J_{50 \rightarrow 49}^{6}=9.97$. This performance index is smaller than the performance index $J_{50 \rightarrow 49}^{3}=$ 14.98 of the control law $\underline{R}_{50 \rightarrow 49}^{3}$ and it is smaller than the performance index $J_{50 \rightarrow 49}^{7}=16.43$ of the control law $\underline{R}_{50 \rightarrow 49}^{7}$. The supervisory control chose a path through the graph the way, that simplex '50' has to be left through the facet that is adjacent to simplex '49', using valve position '6'. The needed continuous input $\underline{u}$ is guaranteed to stay within their upper bounds $\underline{c}_{u}$ and lower bounds $\underline{c}_{l}$

$$
\underline{c}_{l} \leq \underline{u}=\underline{R} \underline{x}+\underline{u}_{0} \leq \underline{c}_{u} .
$$

In figure 7 , the two states, i.e. the levels in tank 1 and 3 , the influx to tank 1 and 3 and the binary state of the discrete inputs for the valves are plotted over time. For non-zero values the valves $V 1, V 2$ and $V 13 u$ are open. If there is a function value for $V 1$ the valve $V 1$ is open. There is no steady state error. The influes $q_{1}$ and $q_{2}$ stay within their bounds of $0<q_{i}<1$. The calculation on a $2.4 \mathrm{GHz}$ Pentium IV for the local control was done in about 20 minutes, using MatLaB R12.1

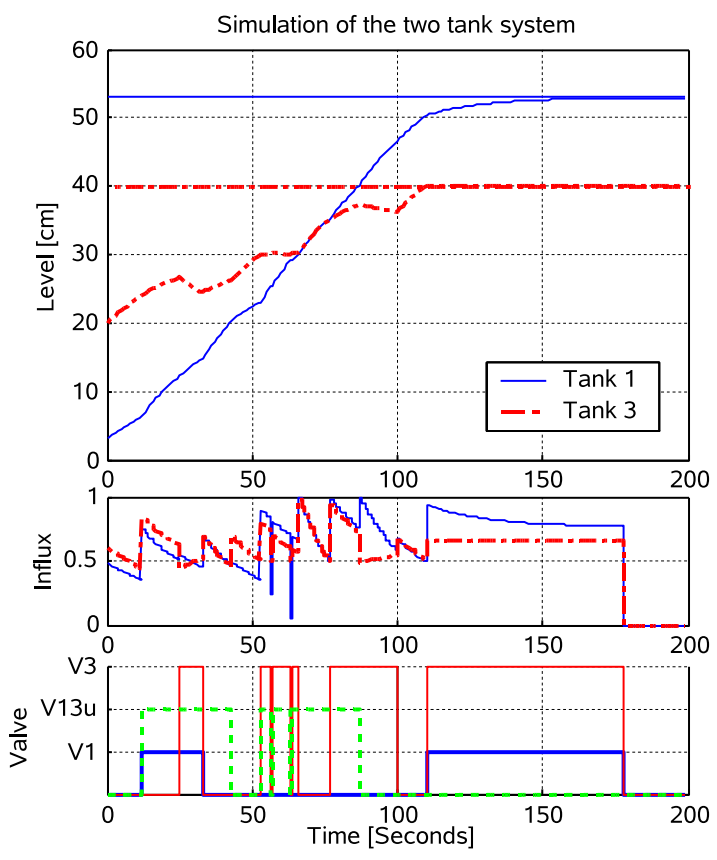

Fig. 7. The level of the two tank system and the binary state of the valves.

\section{CONCLUSION}

A new approach for the design of controllers for piecewise affine hybrid systems was presented.
Based on a discrete-time model, the design of the controller is divided into a local control and a supervisory control. The supervisory control is performed in a graph, done by path planning, the local control is based on choosing system inherent bounds. The resulting control law is an affine feedback law, valid on the corresponding simplex. Linear search algorithms reduce the online computational effort for the control action, determining which control law is active. For that reason the presented method is suitable even for more complex industrial applications.

\section{REFERENCES}

Bemporad, A. and M. Morari (1999). Control of systems integrating logic, dynamics, and contraints. Automatica 35, 407-427.

Borrelli, F. (2002). Discrete Time Constrained Optimal Control. PhD thesis. Eidgenössische Technische Hochschule Zürich.

Branicky, M.S. (1995). Studies in Hybrid Systems: Modeling, Analysis, and Control. PhD thesis. MIT.

Ferrari-Trecate, G. et al. (2001). Identification of piecewise affine and hybrid systems. In: Proceedings of the ACC. CD, Arlington, USA.

Habets, L. and J. van Schuppen (2004). A control problem for affine dynamical systems on a full-dimesnional polytope. Automatica (40), 21-35.

Hodrus, T. and E. Münz (2003). Hybride Phänomene in zeitdiskreter Darstellung. at-Automatisierungstechnik 51(12), 574-582.

Hodrus, T. E., M. Schwarz and V. Krebs (2004). A time discretization method for a class of hybrid systems. NOLCOS Symposium on Nonlinear Control Systems.

Imura, J. (2004). Optimal ccontrol of sampleddata piecewise affine system. Automatica 40, 661-669.

Münz, E. and V. Krebs (2002). Identification of hybrid systems using a priori knowledge. In: Proceedings of the IFAC World Congress. CD, Barcelona, Spain.

Nemhauser, G. L., K. Rinnooy and M. J. Todd (1989). Optimization. Vol. 1 of Handbooks in operations research and management science. Elsevier Science Publishers B.V.. Amsterdam.

Nenninger, Gero Michael (2001). Modellbildung und Analyse hybrider dynamischer Systeme als Grundlage für den Entwurf hybrider Steuerungen. In: Fortschritt-Berichte VDI. Vol. 902. VDI Verlag GmbH. Düsseldorf.

Sontag, E. D. (1981). Nonlinear regulation: The piecewise linear approach. IEEE Transactions on Automatic Control. 